\title{
Synthesis and biological evaluation of cyclic imides incorporating benzenesulfonamide moieties as carbonic anhydrase I, II, IV and IX inhibitors
}

\author{
Alaa A.-M. Abdel-Aziz ${ }^{\mathrm{a}, \mathrm{b}, \mathrm{c}, *}$, Andrea Angeli ${ }^{\mathrm{c}}$, Adel S. El-Azab ${ }^{\mathrm{a}, \mathrm{d}}$, Mohamed A. Abu El-Enin ${ }^{\mathrm{b}}$, \\ Claudiu T. Supuran ${ }^{\mathrm{c}, *}$ \\ a Department of Pharmaceutical Chemistry, College of Pharmacy, King Saud University, Riyadh 11451, Saudi Arabia \\ ${ }^{\mathrm{b}}$ Department of Medicinal Chemistry, Faculty of Pharmacy, University of Mansoura, Mansoura 35516, Egypt \\ ' Università degli Studi di Firenze, NEUROFARBA Dept., Sezione di Scienze Farmaceutiche, Via Ugo Schiff 6, 50019 Sesto Fiorentino, Florence, Italy \\ ${ }^{\mathrm{d}}$ Department of Organic Chemistry, Faculty of Pharmacy, Al-Azhar University, Cairo 11884, Egypt
}

\section{A R T I C L E I N F O}

\section{Article history:}

Received 14 December 2016

Revised 18 January 2017

Accepted 19 January 2017

Available online $\mathrm{xxxx}$

\section{Keywords:}

Cyclic imide

Benzenesulfonamide

Carbonic anhydrase

Inhibitor

\begin{abstract}
A B S T R A C T
A group of cyclic imides was synthesized by reaction of amino-substituted benzenesulfonamides with a series of acid anhydrides such as succinic, maleic, tetrahydrophthalic, pyrazine-2,3-dicarboxylic acid anhydride, and substituted phthalic anhydrides. The synthesized sulfonamides were evaluated as carbonic anhydrase (CA, EC 4.2.1.1) inhibitors against the human (h) isoforms hCA I, II, IV and IX, involved in a variety of diseases among which glaucoma, retinitis pigmentosa, etc. Some of these sulfonamides showed effective inhibitory action (in the nanomolar range) against the cytosolic isoform hCA II and the transmembrane, tumor-associated one hCA IX, making them interesting candidates for preclinical evaluation in glaucoma or various tumors in which the two enzymes are involved. hCA I and IV were on the other hand less inhibited by these sulfonamides, with inhibition constants in the micromolar range.
\end{abstract}

(c) 2017 Elsevier Ltd. All rights reserved.

\section{Introduction}

In previous works from these and other groups, ${ }^{1-11}$ the preparation and biological activity of cyclic imides incorporating primary sulfonamide moieties were investigated. Phthalimides were definitely the privileged such scaffolds, being shown that they may lead to effective inhibitors of the metallo-enzyme carbonic anhydrase (CA, EC 4.2.1.1). ${ }^{2,8-11}$ Furthermore, some substituted-phthalimides derived from aromatic sulfonamides such as sulfanilamide, metanilamide or aminoalkyl-benzenesulfonamides, not only showed low nanomolar activity as CA inhibitors (CAIs), but some of them were also highly isoform-selective inhibitors for several CAs involved in relevant pathologies such as glaucoma, ${ }^{12}$ epilepsy, ${ }^{13}$ obesity, ${ }^{14}$ or cancer. $^{15}$ Indeed, of the 15 different human(h) CA isoforms known to date, many are involved in such and other pathologies, ${ }^{16}$ and finding isoform-selective inhibitors

\footnotetext{
* Corresponding authors at: Università degli Studi di Firenze, NEUROFARBA Dept., Sezione di Scienze Farmaceutiche, Via Ugo Schiff 6, 50019 Sesto Fiorentino, Florence, Italy (A.A.-M. Abdel-Aziz)

E-mail addresses: alaa_moenes@yahoo.com (A.A.-M. Abdel-Aziz), claudiu. supuran@unifi.it (C.T. Supuran).
}

is rather challenging, although many such compounds belonging to various classes of inhibitors were reported by now to possess such properties. ${ }^{17}$ Sulfonamides are in clinical use as CAIs for more than 70 years, primarily as antiglaucoma agents or diuretics. ${ }^{12-17}$

Continuing our interest in sulfonamide CAIs, in this work we report the synthesis and human (h) hCA I, II, IV and IX inhibitory activity of cyclic imides obtained from amino-substituted benzenesulfonamides and a series of acid anhydrides such as succinic, maleic, tetrahydrophthalic, pyrazine-2,3-dicarboxylic acid anhydride, and substituted phthalic anhydrides.

\section{Results and discussion}

\subsection{Drug design and chemistry}

The lead molecules for designing the CAIs synthesized here were the sulfanilamides incorporating phthalimides moieties reported earlier by one of these groups. ${ }^{2,9-11}$ We have shown that reaction of amino-substituted benzenesulfonamides, such as sulfanilamide, 4-aminomethylbenzenesulfonamide or 4-aminoethylbenzenesulfonamide with phthalic anhydride or substituted-phthalic anhydrides leads to the corresponding phthalimides derivatives in 
high yields. ${ }^{2,9-11}$ Furthermore, the new sulfonamides showed effective inhibitory action against human CA isoforms I, II, IV and IX. ${ }^{2,9-11}$

In the present work we have included diverse anhydrides for the preparation of the imides, among which succinic, maleic, tetrahydrophthalic, pyrazine-2,3-dicarboxylic acid anhydride, substituted phthalic anhydrides and norbornene dicarboxylic anhydride. In this way, a wider range of chemical space is being explored in order to investigate the effects of the cyclic imide scaffold on the biological activity of these new sulfonamides.

The preparation of designed imides analogues 1-15 is shown in Schemes 1 and 2. Condensation of acid anhydrides with 4-(2-aminoethyl)benzenesulfonamide in refluxing acetic acid afforded imides 1-12 in good yields (Scheme 1$)^{1-6}$

The remaining compounds, 13-15 (Scheme 2) were prepared in a similar manner, but in this case the norbornene dicarboxylic anhydride has been employed, leading to the tricyclic sulfonamides 13-15. Such scaffolds were not employed earlier for obtaining CAIs.

Compounds 1-15 have been characterized in detail by physicochemical methods which confirmed their structures (see Experimental for details).

\subsection{Carbonic anhydrase inhibition}

The newly synthesized compounds 1-15 were screened for their CA inhibitory activity by a stopped-flow $\mathrm{CO}_{2}$ hydrase assay ${ }^{18}$ against the following isoforms: hCA I, II (cytosolic isoforms involved in glaucoma ${ }^{13}$ and possibly other pathologic states) ${ }^{19}$ and hCA IV (an isoform involved in glaucoma and other eye diseases, such as retinitis pigmentosa), ${ }^{20}$ as well as the transmembrane, tumor-associated hCA IX ${ }^{15,21}$ see Table 1.

The following structure-activity relationships (SARs) are observed and reported for these series of compounds:

(i) The slow cytosolic isoform hCA I was inhibited by all the investigated compounds with inhibition constants ranging between 49.2 and $5121 \mathrm{nM}$. Sulfonamides incorporating tetrahydrophthalimido and tert-butyl phthalimido moieties 3 and 7 showed the best inhibitory activity ( $K_{\mathrm{I}}$ of 49.2 and $95.7 \mathrm{nM}$, respectively). The other scaffolds were less potent and inhibited this isoform in the high nanomolar range $\left(\mathrm{K}_{\mathrm{I}}\right.$ ranging between 179 and $865 \mathrm{nM}$ ). An interesting case was constituted by the isosteric substitution of the chlorine atoms in the phthalimido compounds $\mathbf{9}$ with bromine, in 10, which led to a significant decrease of the inhibitory potency. Considering the monocyclic imides 1, 2 and the bicyclic scaffold 3-15, the inhibition potency against the hCA I did not vary significantly, except for compound $\mathbf{3}$, which was the best inhibitor in the subseries. The linker between the hexachloronorbornene dicarboxylic scaffold and the sulfonamide part of the molecules present in $\mathbf{1 3}$, 14 and 15 also modulated the inhibition profile. The introduction of a methylene chain, as in 14, increased the inhibition potency from $390 \mathrm{nM}$ (13) to $201 \mathrm{nM}$ (14), but a further chain elongation, as in $\mathbf{1 5}$, decreased two times the $\mathrm{K}_{\mathrm{I}}$ for the last compound.

(ii) hCA II, the dominant physiologic isoform, was inhibited by all compounds in the low to high nanomolar range, with $\mathrm{K}_{\mathrm{I}} \mathrm{s}$ of 5-693 nM. The tetrahydrophthalimido derivative 3

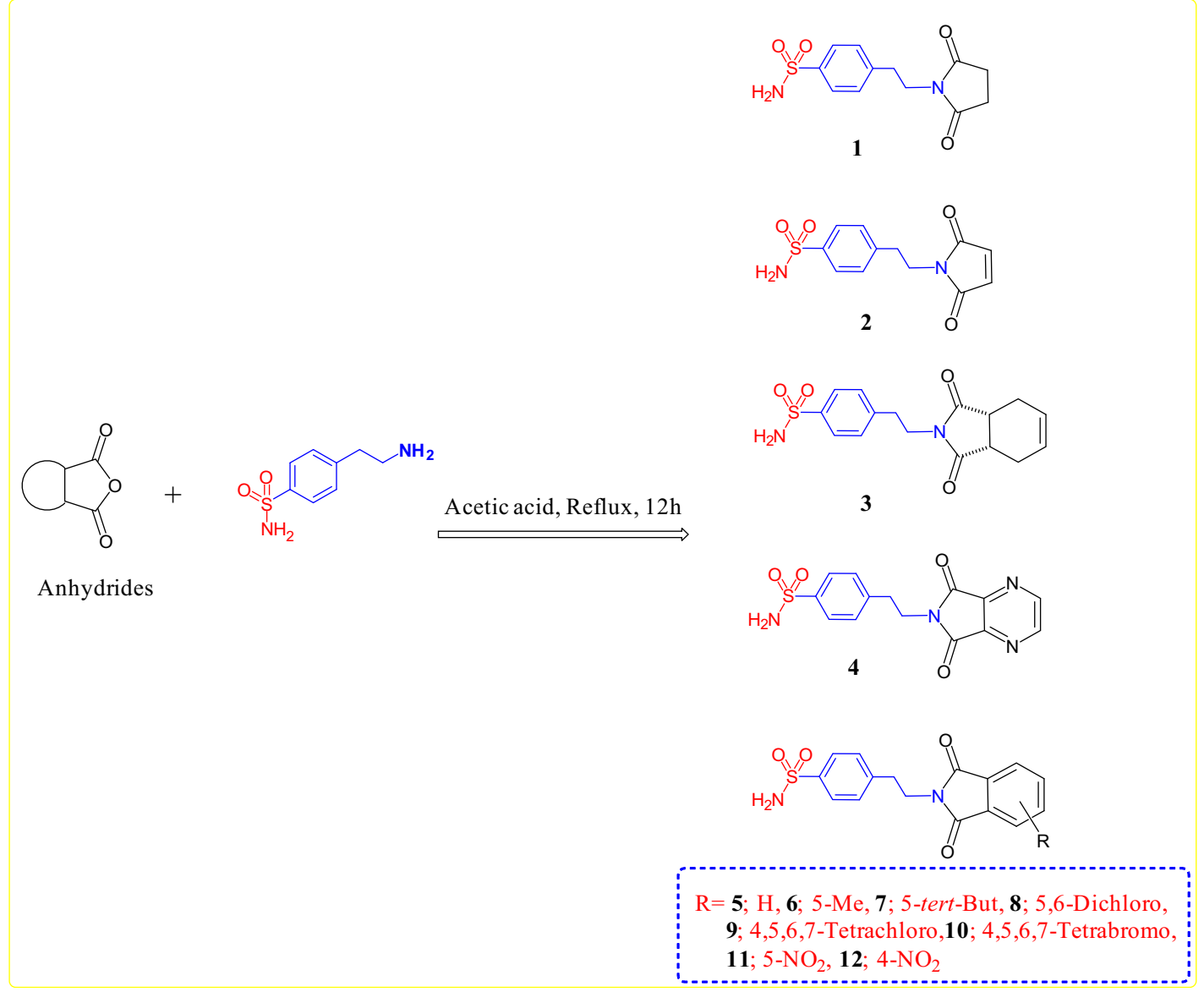

Scheme 1. Synthesis of the designed cyclic imide derivatives 1-12. 


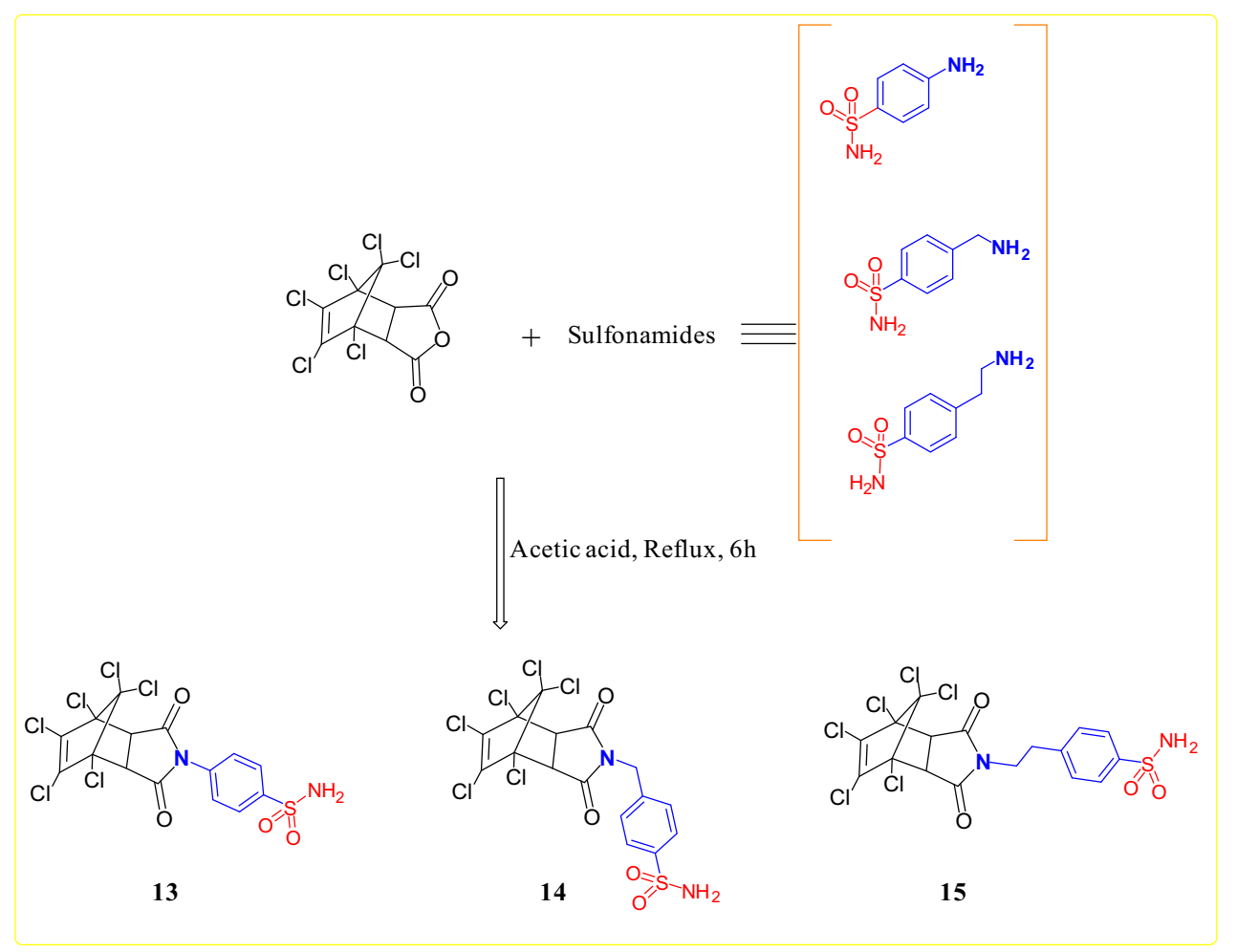

Scheme 2. Synthesis of the designed hexachloro norbornene dicarboxylic imides derivatives 13-15.

Table 1

In vitro CA I, II, IV and IX inhibition with compounds 1-15 and acetazolamide as standard, by a stopped-flow, $\mathrm{CO}_{2}$ hydrase assay. ${ }^{18}$

\begin{tabular}{|c|c|c|c|c|}
\hline \multirow[t]{2}{*}{ Compound } & \multicolumn{4}{|c|}{$\mathrm{K}_{\mathrm{I}}(\mathrm{nM})^{\mathrm{a}}$} \\
\hline & hCA I & hCA II & hCA IV & hCA IX \\
\hline 1 & 446 & 73.6 & 9578 & 296 \\
\hline 2 & 617 & 84.5 & 4849 & 274 \\
\hline 3 & 49.2 & 5.0 & 973 & 296 \\
\hline 4 & 441 & 426 & 9380 & 4188 \\
\hline 5 & 491 & 49.4 & 5461 & 296 \\
\hline 6 & 425 & 60.5 & 2925 & 390 \\
\hline 7 & 95.7 & 89.9 & 9391 & 459 \\
\hline 8 & 435 & 79.0 & 9358 & 428 \\
\hline 9 & 562 & 68.1 & 9232 & 284 \\
\hline 10 & 5121 & 693 & 8839 & 2389 \\
\hline 11 & 865 & 140 & 8175 & 1636 \\
\hline 12 & 179 & 85.4 & 8517 & 2667 \\
\hline 13 & 390 & 41.5 & 83.0 & 13.7 \\
\hline 14 & 201 & 54.6 & 912 & 16.9 \\
\hline 15 & 484 & 23.2 & 6764 & 113.6 \\
\hline AAZ & 250 & 12.1 & 74 & 25.2 \\
\hline
\end{tabular}

${ }^{a}$ Mean from 3 different assays, by a stopped flow technique (errors were in the range of $\pm 5-10 \%$ of the reported values).

showed a very good inhibition activity against this isoform, with a $\mathrm{K}_{\mathrm{I}}$ of $5 \mathrm{nM}$. The replacement of the monocyclic imido scaffold with a bicyclic one did not have a significant effect on the inhibition profiles of these sulfonamides (Table 1). Only in the case of the pyrazine containing sulfonamide $\mathbf{4}$, a decreased inhibitory potency was observed compared to the other members of the series, this compound being one of the weakest hCA II inhibitor ( $\mathrm{K}_{\mathrm{I}}$ of $\left.426 \mathrm{nM}\right)$. The substitution on the phthalimido scaffold in compounds 5-12 did not lead to significant changes in the inhibition potency, except for the tetrabromo-substituted compound 10, which was a medium potency - weak hCA II inhibitor $\left(\mathrm{K}_{\mathrm{I}} 693 \mathrm{nM}\right)$. Furthermore, the substitution of the nitro group from position 5 of compound 11 to position 4 (12), increased nearly two times the potency of the corresponding sulfonamide against this. Unlike the other cytosolic isoform, the introduction of a linker in the norborneneimido derivatives 13-15 had diverse effects on the inhibition profile: a decrease of the potency was observed for a methylene linker $\left(13\right.$, a $K_{I}$ of $41.5 \mathrm{nM}$ whereas 14 had a $K_{I}$ of $54.6 \mathrm{nM}$ ), whereas a further chain elongation, as in $\mathbf{1 5}$, led to an increase of the potency with a $\mathrm{K}_{\mathrm{I}}$ of $23.2 \mathrm{nM}$ for the last compound.

(iii) The membrane-bound hCA IV was inhibited by most of compounds investigated here in the micromolar range. The monocyclic imido derivatives $\mathbf{1}$ and $\mathbf{2}$ showed $\mathrm{K}_{\mathrm{I}} \mathrm{S}$ in the range of $4.85-9.57 \mu \mathrm{M}$. The maleimido scaffold present in $\mathbf{3}$ led to a compound which was two times more potent than the succinimido analog $\mathbf{1}$. Also for this isoform, the groups 
present on the phthalimido scaffold did not influence significantly the inhibition profile. The compounds incorporating hexachloronorbornene dicarboxylic scaffolds, 13-15, showed a very interesting inhibition profile. The linker between the imido scaffold and the sulfonamide head was crucial for the inhibition potency. A direct correlation between the length of the linker and the inhibition constant was observed: 13 showed a nanomolar inhibition constant $\left(\mathrm{K}_{\mathrm{I}} 83 \mathrm{nM}\right)$, whereas an increase in the linker length to a methylene or an ethylene group led to a drastic decrease of potency, to the micromolar range ( $K_{\mathrm{I}}$ of $912 \mathrm{nM}$ for compound 14 and $K_{I}$ of $6764 \mathrm{nM}$ for 15 ).

(iv) hCA IX, the tumor-associated isoform, was effectively inhibited by many of the compounds reported here, in high nanomolar range except for compounds 13 and 14 which were active in the low nanomolar range. The monocyclic imido derivatives $\mathbf{1}$ and $\mathbf{2}$ had nearly the same inhibition profile with a $K_{I}$ of $296 \mathrm{nM}$ and $274 \mathrm{nM}$, respectively. The phthalimido scaffold present in 5-12 led to high nanomolar hCA IX inhibitors, except for nitro-substituted compounds 11 and 12. For these compounds the potency has decreased to the micromolar range, with $\mathrm{K}_{\mathrm{I}}$ of $1636 \mathrm{nM}$ and $2667 \mathrm{nM}$, respectively. Moreover, the hexachloro-norbornene scaffold present in 13-15, led to an interesting SAR for the inhibition of this isoform. Compounds $\mathbf{1 3}$ and $\mathbf{1 4}$ incorporating no linker or a methylene linker, exhibited low nanomolar $\mathrm{K}_{\mathrm{I}} \mathrm{S}$ whereas the derivative with a longer chain $\mathbf{1 5}$, as for isoform hCA IV, led to a marked decreased in the inhibition potency (Table 1).

\section{Conclusions}

We investigated a series of cyclic imides as CA inhibitors, continuing our research in this field of enzyme modulators. The compounds were synthesized by reaction of amino-substituted benzenesulfonamides with a series of dicarboxylic acid anhydrides such as succinic, maleic, tetrahydrophthalic, pyrazine-2,3-dicarboxylic acid anhydride, as well as substituted phthalic anhydrides. The synthesized sulfonamides were evaluated for the inhibition of isoforms hCA I, II, IV and IX, involved in a variety of diseases among which glaucoma, retinitis pigmentosa, tumors, etc. They showed effective inhibitory action against the cytosolic isoform hCA II and the transmembrane, tumor-associated one hCA IX, making them interesting candidates for preclinical evaluation in glaucoma or various tumors in which the two enzymes are involved. hCA I and IV were on the other hand less inhibited by these sulfonamides.

\section{Experimental part}

\subsection{Chemistry}

Melting points (uncorrected) were recorded on Barnstead 9100 Electrothermal melting apparatus. IR spectra were recorded on a FT-IR Perkin-Elmer spectrometer. ${ }^{1} \mathrm{H}$ NMR and ${ }^{13} \mathrm{C}$ NMR were recorded in DMSO- $d_{6}$ on Bruker 500 and $125 \mathrm{MHz}$ instrument, respectively, using TMS as internal standard (chemical shifts in $\delta$ ppm). Mass spectra were recorded on a Agilent 6320 Ion Trap mass spectrometers. Elemental analysis was carried out for $\mathrm{C}, \mathrm{H}$ and $\mathrm{N}$ at the Research Centre of College of Pharmacy, King Saud University and the results are within $\pm 0.4 \%$ of the theoretical values. Compounds 2, 5, 9, 10 and 11 were prepared according to their reported procedure. $^{7-11}$
4.1.1. General procedure for the synthesis of cyclic imides $\mathbf{1}, \mathbf{3}, \mathbf{4}, \mathbf{6}-\mathbf{8}$, 12

A mixture of 4-(2-aminoethyl)benzenesulfonamide ( $0.5 \mathrm{~g}$, $2.5 \mathrm{mmol})$, anhydrous sodium acetate $(0.41 \mathrm{~g}, 5.0 \mathrm{mmol})$ and an acid anhydride $(2.5 \mathrm{mmol})$ was stirred in glacial acetic acid $(15 \mathrm{~mL})$. The mixture was heated under reflux for $12 \mathrm{~h}$ and the reaction was monitored with the help of TLC. After completion of reaction the solvent was removed under reduced pressure and the crude solid obtained was washed with water, dried and recrystallised from an appropriate solvent.

\subsubsection{4-(2-(2,5-Dioxopyrrolidin-1-yl)ethyl)benzenesulfonamide (1).}

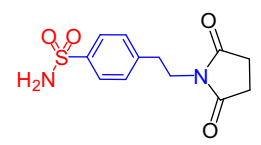

White crystals, M.p. $243-245^{\circ}, 95 \%$ yield $\left(\mathrm{CH}_{3} \mathrm{OH}\right)$; IR $(\mathrm{KBr}$, $\left.\mathrm{cm}^{-1}\right) v: 3496(\mathrm{NH}), 1795,1725(\mathrm{C}=\mathrm{O}), 1382,1194(\mathrm{O}=\mathrm{S}=\mathrm{O}) ;{ }^{1} \mathrm{H}$ $\operatorname{NMR}\left(500 \mathrm{MHz}, \mathrm{CDCl}_{3} / \mathrm{DMSO}_{-} \mathrm{d}_{6}\right): \delta 2.60(\mathrm{~s}, 4 \mathrm{H}), 2.85-2.88(\mathrm{t}, 2 \mathrm{H}$, $J=7.5 \mathrm{~Hz}), 3.60-3.63(\mathrm{t}, 2 \mathrm{H}, J=7.5 \mathrm{~Hz}), 7.28(\mathrm{~s}, 1 \mathrm{H}), 7.35-7.37(\mathrm{~d}$, $2 \mathrm{H}, J=8.0 \mathrm{~Hz}), 7.74-7.76(\mathrm{~d}, 2 \mathrm{H}, J=8.0 \mathrm{~Hz}), 8.22-8.23(\mathrm{t}, 1 \mathrm{H}$, $J=1.5 \mathrm{~Hz}) ;{ }^{13} \mathrm{C}$ NMR $\left(125 \mathrm{MHz}, \mathrm{CDCl}_{3} / \mathrm{DMSO}^{-d_{6}}\right): \delta 28.37,33.15$, $39.21,126.28,129.47,142.76,142.78,177.74 ; \mathrm{C}_{12} \mathrm{H}_{14} \mathrm{~N}_{2} \mathrm{O}_{4} \mathrm{~S}: \mathrm{m} / \mathrm{z}$ (281.99).

4.1.1.2. 4-(2-(1,3-Dioxo-1,3,3a,4,7,7a-hexahydro-2H-isoindol-2-yl) ethyl)benzenesulfonamide (3).

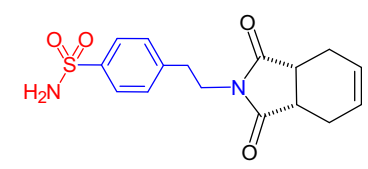

White crystals, M.p. $196-198^{\circ}, 91 \%$ yield $\left(\mathrm{CH}_{3} \mathrm{OH}\right)$; IR $(\mathrm{KBr}$, $\left.\mathrm{cm}^{-1}\right) v: 3230(\mathrm{NH}), 1767,1699(\mathrm{C}=\mathrm{O}), 1333,1179(\mathrm{O}=\mathrm{S}=\mathrm{O}) ;{ }^{1} \mathrm{H}$ NMR (500 MHz, $\mathrm{CDCl}_{3} / \mathrm{DMSO}_{-} \mathrm{d}_{6}$ ): $\delta$ 2.11-2.15 (m, 2H), 2.34-2.37 $(\mathrm{d}, 2 \mathrm{H}, J=13.5 \mathrm{~Hz}), 2.83-2.86(\mathrm{t}, 2 \mathrm{H}, J=7.5 \mathrm{~Hz}), 3.04-3.05(\mathrm{t}, 2 \mathrm{H}$, $J=3.0 \mathrm{~Hz}), 3.61-3.64(\mathrm{t}, 2 \mathrm{H}, J=7.0 \mathrm{~Hz}), 5.73-5.77(\mathrm{~m}, 2 \mathrm{H}), 7.21(\mathrm{~s}$, $2 \mathrm{H}), 7.27-7.29$ (d, $2 \mathrm{H}, J=8.0 \mathrm{~Hz}), 7.72-7.74(\mathrm{~d}, 2 \mathrm{H}, \mathrm{J}=8.5 \mathrm{~Hz}) ;{ }^{13} \mathrm{C}$ NMR (125 MHz, $\left.\mathrm{CDCl}_{3} / \mathrm{DMSO}-d_{6}\right): \delta 25.33,33.09,38.86,42.74$, $127.08,129.33,142.30,142.42,142.78,179.94 ; \mathrm{C}_{16} \mathrm{H}_{18} \mathrm{~N}_{2} \mathrm{O}_{4} \mathrm{~S}: m / z$ (333.70).

4.1.1.3. 4-(2-(5,7-Dioxo-5,7-dihydro-6H-pyrrolo[3,4-b]pyrazin-6-yl) ethyl)benzenesulfonamide (4).

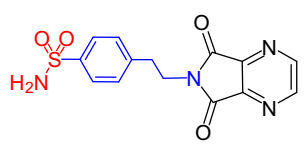

Gray powder, M.p. $120-122^{\circ}$, $76 \%$ yield $\left(\mathrm{CH}_{3} \mathrm{OH} / \mathrm{CH}_{2} \mathrm{Cl}_{2}\right)$; IR $\left(\mathrm{KBr}, \mathrm{cm}^{-1}\right) v: 3430(\mathrm{NH}), 1782,1707(\mathrm{C}=\mathrm{O}), 1318,1129(\mathrm{O}=\mathrm{S}=\mathrm{O})$; ${ }^{1} \mathrm{H}$ NMR (500 MHz, DMSO- $\left.d_{6}\right): \delta 2.76-2.79(\mathrm{t}, 2 \mathrm{H}, J=7.0 \mathrm{~Hz}), 3.27-$ $3.03(\mathrm{t}, 2 \mathrm{H}, J=7.0 \mathrm{~Hz}), 7.38-7.41(\mathrm{t}, 3 \mathrm{H}, J=8.0 \mathrm{~Hz}), 7.74-7.75(\mathrm{~d}$, $3 \mathrm{H}, J=8.5 \mathrm{~Hz}), 8.01-8.03(\mathrm{t}, 1 \mathrm{H}, J=5.0 \mathrm{~Hz}), 9.11(\mathrm{~s}, 1 \mathrm{H}) ;{ }^{13} \mathrm{C} \mathrm{NMR}$ $\left(125 \mathrm{MHz}, \mathrm{DMSO}-d_{6}\right): \delta 35.28,38.65,126.13,129.54,142.47$, 144.22, 149.01, 169.77; $\mathrm{C}_{14} \mathrm{H}_{12} \mathrm{~N}_{4} \mathrm{O}_{4} \mathrm{~S}: \mathrm{m} / z$ (331.76). 
4.1.1.4. 4-(2-(5-Methyl-1,3-dioxoisoindolin-2-yl)ethyl)benzenesulfonamide (6).

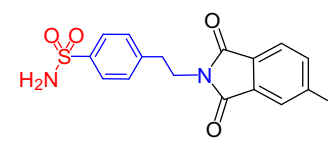

White crystals, Mp 221-222 ${ }^{\circ}$, 93\% yield $\left(\mathrm{CH}_{3} \mathrm{OH} / \mathrm{CH}_{2} \mathrm{Cl}_{2}\right)$; IR $\left(\mathrm{KBr}, \mathrm{cm}^{-1}\right) v: 3466(\mathrm{NH}), 1767,1722(\mathrm{C}=\mathrm{O}), 1300,1131(\mathrm{O}=\mathrm{S}=\mathrm{O})$; ${ }^{1} \mathrm{H} \mathrm{NMR}\left(500 \mathrm{MHz}, \mathrm{CDCl}_{3} / \mathrm{DMSO}_{-} \mathrm{d}_{6}\right): \delta 2.43-2.45(\mathrm{~d}, 3 \mathrm{H}, J=9.0 \mathrm{~Hz})$, $2.96-2.99(\mathrm{t}, 2 \mathrm{H}, J=7.0 \mathrm{~Hz}), 3.79-3.84(\mathrm{q}, 2 \mathrm{H}, J=8.0,7.0 \mathrm{~Hz}), 6.97$ $(\mathrm{s}, 2 \mathrm{H}), 7.26-7.00(\mathrm{t}, 2 \mathrm{H}, J=8.5 \mathrm{~Hz}), 7.46-7.49(\mathrm{t}, 1 \mathrm{H}, J=7.5 \mathrm{~Hz})$, $7.53-7.54(\mathrm{~d}, 1 \mathrm{H}, J=7.5 \mathrm{~Hz}), 7.60-7.63(\mathrm{t}, 1 \mathrm{H}, J=7.5 \mathrm{~Hz}), 7.71-$ $7.74(\mathrm{t}, 2 \mathrm{H}, J=7.5 \mathrm{~Hz}) ;{ }^{13} \mathrm{C}$ NMR $\left(125 \mathrm{MHz}, \mathrm{CDCl}_{3} / \mathrm{DMSO}_{-} \mathrm{d}_{6}\right): \delta$ $22.00,34.19,38.62,123.17,123.80,126.30,129.19,132.20$, $134.76,142.41,142.50,145.43,167.91,168.02 ; \mathrm{C}_{17} \mathrm{H}_{16} \mathrm{~N}_{2} \mathrm{O}_{4} \mathrm{~S}: m / z$ (344.07).

4.1.1.5. 4-(2-(5-(tert-Butyl)-1,3-dioxoisoindolin-2-yl)ethyl)benzenesulfonamide (7).

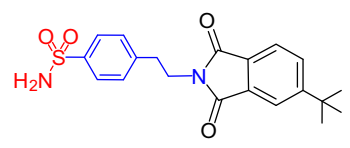

White powder, M.p. $160-162^{\circ}, 89 \%$ yield $\left(\mathrm{CH}_{3} \mathrm{OH} / \mathrm{CH}_{2} \mathrm{Cl}_{2}\right)$; IR $\left(\mathrm{KBr}, \mathrm{cm}^{-1}\right) v$ : 3493, $3095(\mathrm{NH}), 1780,1727(\mathrm{C}=\mathrm{O}), 1360,1236$ $(\mathrm{O}=\mathrm{S}=\mathrm{O}) ;{ }^{1} \mathrm{H}$ NMR $\left(500 \mathrm{MHz}, \mathrm{DMSO}-d_{6}\right): \delta 1.32(\mathrm{~s}, 9 \mathrm{H}), 2.96-$ $2.99(\mathrm{t}, 2 \mathrm{H}, J=7.0 \mathrm{~Hz}), 3.82-3.85(\mathrm{t}, 2 \mathrm{H}, J=7.5 \mathrm{~Hz}), 6.94(\mathrm{~s}, 2 \mathrm{H})$, $7.28-7.30(\mathrm{~d}, 2 \mathrm{H}, J=8.0 \mathrm{~Hz}), 7.69-7.70(\mathrm{t}, 2 \mathrm{H}, J=7.0 \mathrm{~Hz}), 7.73-$ $7.55(\mathrm{t}, 3 \mathrm{H}, J=8.5 \mathrm{~Hz}) ;{ }^{13} \mathrm{C}$ NMR $\left(125 \mathrm{MHz}\right.$, DMSO-d $\left.d_{6}\right): \delta 31.17$, 34.22 , 35.73, 38.61, 120.30, 123.13, 126.32, 129.18, 131.22, $132.07,142.39,142.46,158.56,167.88,168.26 ; \mathrm{C}_{20} \mathrm{H}_{22} \mathrm{~N}_{2} \mathrm{O}_{4} \mathrm{~S}: m / z$ (387.15).

4.1.1.6. 4-(2-(5,6-Dichloro-1,3-dioxoisoindolin-2-yl)ethyl)benzenesulfonamide (8).

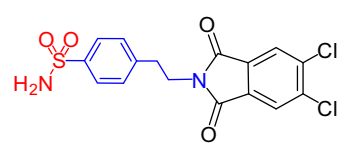

White crystals, Mp 295-296, $91 \%$ yield $\left(\mathrm{CH}_{3} \mathrm{OH}\right)$; IR $\left(\mathrm{KBr}, \mathrm{cm}^{-1}\right)$ $v$ : 3469, $3369(\mathrm{NH}), 1727,1670(\mathrm{C}=\mathrm{O}), 1366,1167(\mathrm{O}=\mathrm{S}=\mathrm{O}) ;{ }^{1} \mathrm{H}$ NMR (500 MHz, DMSO- $\left.d_{6}\right): \delta 2.99-3.02(\mathrm{t}, 2 \mathrm{H}, J=7.5 \mathrm{~Hz}), 3.83-$ $3.86(\mathrm{t}, 2 \mathrm{H}, J=7.5 \mathrm{~Hz}), 7.31(\mathrm{~s}, 2 \mathrm{H}), 7.40-7.42(\mathrm{~d}, 2 \mathrm{H}, J=8.0 \mathrm{~Hz})$, $7.71-7.73(\mathrm{t}, 2 \mathrm{H}, J=8.5 \mathrm{~Hz}), 8.15-8.16(\mathrm{t}, 2 \mathrm{H}, J=8.5 \mathrm{~Hz}) ;{ }^{13} \mathrm{C} \mathrm{NMR}$ $\left(125 \mathrm{MHz}, \mathrm{DMSO}-d_{6}\right): \delta 33.78,39.34,125.74,126.26,129.67$, 131.88, 137.81, 142.80, 166.32; $\mathrm{C}_{16} \mathrm{H}_{12} \mathrm{Cl}_{2} \mathrm{~N}_{2} \mathrm{O}_{4} \mathrm{~S}: \mathrm{m} / z$ (398.60).

4.1.1.7. 4-(2-(4-Nitro-1,3-dioxoisoindolin-2-yl)ethyl)benzenesulfonamide (12).
Yellow powder, Mp 238-240, $86 \%$ yield $\left(\mathrm{CH}_{3} \mathrm{OH} / \mathrm{CH}_{2} \mathrm{Cl}_{2}\right)$; IR $\left(\mathrm{KBr}, \mathrm{cm}^{-1}\right) v$ : $3485(\mathrm{NH}), 1779,1732(\mathrm{C}=\mathrm{O}), 1362,1228(\mathrm{O}=\mathrm{S}=\mathrm{O})$; ${ }^{1} \mathrm{H}$ NMR (500 MHz, DMSO- $\left.d_{6}\right): \delta 2.99-3.02(\mathrm{t}, 2 \mathrm{H}, J=6.0 \mathrm{~Hz}), 3.83-$ $3.86(\mathrm{t}, 2 \mathrm{H}, J=7.5 \mathrm{~Hz}), 7.32(\mathrm{~s}, 2 \mathrm{H}), 7.44-7.45$ (d, $2 \mathrm{H}, J=8.5 \mathrm{~Hz})$, 7.73-7.75 (t, $2 \mathrm{H}, J=6.0 \mathrm{~Hz}), 8.05-8.07(\mathrm{t}, 1 \mathrm{H}, J=5.0 \mathrm{~Hz}), 8.15-$ $8.16(\mathrm{t}, 1 \mathrm{H}, J=5.5 \mathrm{~Hz}), 8.28-8.29(\mathrm{t}, 1 \mathrm{H}, J=5.0 \mathrm{~Hz}) ;{ }^{13} \mathrm{C}$ NMR $\left(125 \mathrm{MHz}, \mathrm{DMSO}-d_{6}\right): \delta 33.72,39.44,123.44,126.29,127.32$, $128.80,129.65,133.92,136.70,142.82,142.89,144.76,163.65$, 166.19; $\mathrm{C}_{16} \mathrm{H}_{13} \mathrm{~N}_{3} \mathrm{O}_{6} \mathrm{~S}: \mathrm{m} / z$ (374.54).

4.1.2. General procedure for the synthesis of cyclic imides 13-15

A mixture of benzenesulfonamide $(2.5 \mathrm{mmol})$, anhydrous sodium acetate $(0.41 \mathrm{~g}, 5.0 \mathrm{mmol})$ and $4,5,6,7,8,8$-hexachloro3a,4,7,7a-tetrahydro-4,7-methanoisobenzofuran-1,3-dione $(0.927 \mathrm{~g}, 2.5 \mathrm{mmol})$ were stirred in glacial acetic acid $(15 \mathrm{~mL})$ and heated under reflux for $6 \mathrm{~h}$. After the evaporation of the solvent under reduced pressure, the precipitate obtained was washed with water, dried and re-crystallised from an appropriate solvent.

4.1.2.1. 4-(4,5,6,7,8,8-Hexachloro-1,3-dioxo-1,3,3a,4,7,7a-hexahydro2H-4,7-methanoisoindol-2-yl)benzenesulfonamide (13).

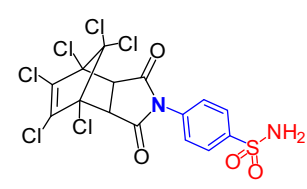

White powder, M.p. > 350, $95 \%$ yield $\left(\mathrm{CH}_{3} \mathrm{OH}\right) ; \mathrm{IR}\left(\mathrm{KBr}, \mathrm{cm}^{-1}\right) v$ : 3370, $3250(\mathrm{NH}), 1769,1708(\mathrm{C}=\mathrm{O}), 1388,1144(\mathrm{O}=\mathrm{S}=\mathrm{O}) ;{ }^{1} \mathrm{H}$ NMR $\left(500 \mathrm{MHz}, \mathrm{DMSO}-d_{6}\right): \delta 4.28(\mathrm{~s}, 2 \mathrm{H}), 7.34-7.35(\mathrm{~d}, 2 \mathrm{H}, J=8.5 \mathrm{~Hz}$ ), $7.52(\mathrm{~s}, 2 \mathrm{H}), 8.00-8.01(\mathrm{~d}, 2 \mathrm{H}, J=8.5 \mathrm{~Hz}) ;{ }^{13} \mathrm{C}$ NMR $(125 \mathrm{MHz}$, DMSO- $\left.d_{6}\right): \delta 52.70,79.44,104.15,127.52,131.12,134.07,145.07$, 169.85; $\mathrm{C}_{15} \mathrm{H}_{8} \mathrm{Cl}_{6} \mathrm{~N}_{2} \mathrm{O}_{4} \mathrm{~S}: m / z$ (526.43).

4.1.2.2. 4-((4,5,6,7,8,8-Hexachloro-1,3-dioxo-1,3,3a,4,7,7a-hexahydro-2H-4,7-methanoisoindol-2-yl)methyl)benzenesulfonamide (14).

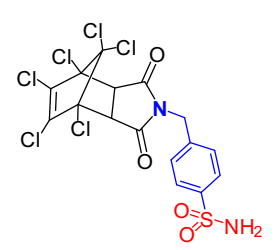

White powder, M.p. $201-203^{\circ}, 87 \%$ yield $\left(\mathrm{CH}_{3} \mathrm{OH} / \mathrm{CH}_{2} \mathrm{Cl}_{2}\right)$; IR $\left(\mathrm{KBr}, \mathrm{cm}^{-1}\right) v: 3307,3229(\mathrm{NH}), 1759,1711(\mathrm{C}=\mathrm{O}), 1369,1180$ $(\mathrm{O}=\mathrm{S}=\mathrm{O}) ;{ }^{1} \mathrm{H}$ NMR $\left(500 \mathrm{MHz}, \mathrm{CDCl}_{3} / \mathrm{DMSO}_{-} \mathrm{d}_{6}\right): \delta 3.95(\mathrm{~s}, 2 \mathrm{H})$, $4.52(\mathrm{~s}, 2 \mathrm{H}), 6.89(\mathrm{~s}, 2 \mathrm{H}), 7.36-7.38(\mathrm{~d}, 2 \mathrm{H}, J=8.5 \mathrm{~Hz}), 7.77-7.78$ $(\mathrm{d}, 2 \mathrm{H}, J=8.0 \mathrm{~Hz}) ;{ }^{13} \mathrm{C}$ NMR $\left(125 \mathrm{MHz}, \mathrm{CDCl}_{3} /\right.$ DMSO-d $\left.\mathrm{d}_{6}\right): \delta 42.49$, 52.09, 79.43, 104.37, 126.39, 129.74, 130.58, 138.23, 143.87, $170.05 ; \mathrm{C}_{16} \mathrm{H}_{10} \mathrm{Cl}_{6} \mathrm{~N}_{2} \mathrm{O}_{4} \mathrm{~S}: \mathrm{m} / \mathrm{z}(539.50)$.

4.1.2.3. 4-(2-(4,5,6,7,8,8-Hexachloro-1,3-dioxo-1,3,3a,4,7,7a-hexahydro-2H-4,7-methanoisoindol-2-yl)ethyl)benzenesulfonamide (15).
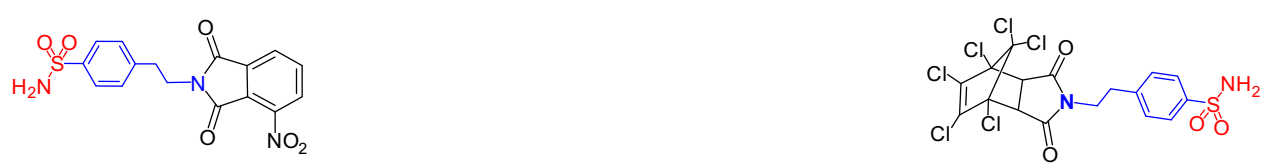
White powder, M.p. 226-228 ${ }^{\circ}, 83 \%$ yield $\left(\mathrm{CH}_{3} \mathrm{OH} / \mathrm{CH}_{2} \mathrm{Cl}_{2}\right)$; IR $\left(\mathrm{KBr}, \mathrm{cm}^{-1}\right) v: 3323,3213(\mathrm{NH}), 1751,1714(\mathrm{C}=\mathrm{O}), 1373,1165$ $(\mathrm{O}=\mathrm{S}=\mathrm{O}) ;{ }^{1} \mathrm{H}$ NMR $\left(500 \mathrm{MHz}, \mathrm{CDCl}_{3} / \mathrm{DMSO}_{-} \mathrm{d}_{6}\right): \delta 2.71-2.74(\mathrm{t}$, $2 \mathrm{H}, J=10.0 \mathrm{~Hz}), 3.55-3.58(\mathrm{q}, 2 \mathrm{H}, J=10.0,5.5 \mathrm{~Hz}), 4.01-4.02(\mathrm{~d}$, $2 \mathrm{H}, J=3.5 \mathrm{~Hz}), 7.17(\mathrm{~s}, 2 \mathrm{H}), 7.33-7.34(\mathrm{t}, 2 \mathrm{H}, J=4.0 \mathrm{~Hz}), 7.76-$ 7.78 (q, $2 \mathrm{H}, J=8.5,4.5 \mathrm{~Hz}) ;{ }^{13} \mathrm{C}$ NMR $\left(125 \mathrm{MHz}, \mathrm{CDCl}_{3} / \mathrm{DMSO}_{-} \mathrm{d}_{6}\right)$ : $\delta$ 33.42, 39.60, 52.17, 79.26, 104.36, 126.41, 129.20, 130.71, 141.39, 143.04, 170.38; $\mathrm{C}_{17} \mathrm{H}_{12} \mathrm{Cl}_{6} \mathrm{~N}_{2} \mathrm{O}_{4} \mathrm{~S}: m / z$ (552.19).

\subsection{Carbonic anhydrase inhibition}

An SX.18MV-R Applied Photophysics (Oxford, UK) stopped-flow instrument has been used to assay the catalytic/inhibition of various CA isozymes. ${ }^{18}$ Phenol Red (at a concentration of $0.2 \mathrm{mM}$ ) has been used as indicator, working at the absorbance maximum of $557 \mathrm{~nm}$, with $10 \mathrm{mM}$ Hepes ( $\mathrm{pH} 7.4$ ) as buffer, $0.1 \mathrm{M} \mathrm{Na}_{2} \mathrm{SO}_{4}$ or $\mathrm{NaClO}_{4}$ (for maintaining constant the ionic strength; these anions are not inhibitory in the used concentration), ${ }^{22}$ following the CAcatalyzed $\mathrm{CO}_{2}$ hydration reaction for a period of 5-10 s. Saturated $\mathrm{CO}_{2}$ solutions in water at $25{ }^{\circ} \mathrm{C}$ were used as substrate. Stock solutions of inhibitors were prepared at a concentration of $10 \mathrm{mM}$ (in DMSO-water $1: 1, \mathrm{v} / \mathrm{v}$ ) and dilutions up to $0.01 \mathrm{nM}$ done with the assay buffer mentioned above. At least 7 different inhibitor concentrations have been used for measuring the inhibition constant. Inhibitor and enzyme solutions were preincubated together for $10 \mathrm{~min}$ at room temperature prior to assay, in order to allow for the formation of the E-I complex. Triplicate experiments were done for each inhibitor concentration, and the values reported throughout the paper are the mean of such results. The inhibition constants were obtained by non-linear least-squares methods using the Cheng-Prusoff equation, as reported earlier, ${ }^{11}$ and represent the mean from at least three different determinations. All CA isozymes used here were recombinant proteins obtained as reported earlier by our group. ${ }^{23-26}$

\section{Acknowledgments}

The authors express their appreciation to the Deanship of Scientific Research at King Saud University for funding the work through the research group project No. RGP-163.

\section{References}

1. Alanazi AM, El-Azab AS, Al-Suwaidan IA, et al. Eur J Med Chem. 2015;92:115.

2. (a) Abdel-Aziz AA-M, El-Azab AS, Ceruso M, Supuran CT. Bioorg Med Chem Lett. 2014;24:5185;

(b) El-Azab AS, Abdel-Aziz AA-M, Ayyad RR, Ceruso M, Supuran CT. Bioorg Med Chem. 2016;24:20.

3. Al-Suwaidan IA, Alanazi AM, El-Azab AS, et al. Bioorg Med Chem Lett. 2013;23:2601.

4. Abdel-Aziz AA-M, ElTahir KEH, Asiri YA. Eur J Med Chem. 2011;46:1648.

5. Abdel-Aziz AA-M, El-Azab AS, Attia SM, Al-Obaid AM, Al-Omar MA, El-Subbagh HI. Eur J Med Chem. 2011;46:4324.

6. Abdel-Aziz AA-M. Eur J Med Chem. 2007;42:612.

7. Liu G-N, Luo R-H, Zhang X-J, et al. Med Chem. 2014;4:573.

8. Scozzafava A, Mincione F, Menabuoni L, Supuran CT. Drug Des Discov. 2001; 17:337.

9. Sethi KK, Verma SM, Tanc M, et al. Bioorg Med Chem. 2014;22:1586.

10. Sethi KK, Verma SM, Tanc M, Carta F, Supuran CT. Bioorg Med Chem. 2013;21:5168.

11. Sethi KK, Vullo D, Verma SM, Tanc M, Carta F, Supuran CT. Bioorg Med Chem. 2013;21:5973.
12. (a) De Simone G, Alterio V, Supuran CT. Expert Opin Drug Discov. 2013;8:793; (b) Masini E, Carta F, Scozzafava A, Supuran CT. Expert Opin Ther Pat 2013;23:705;

(c) Supuran CT. Bioorg Med Chem Lett. 2010;20:3467;

(d) Supuran CT. Expert Opin Drug Discov. 2017;12:61;

(e) Supuran CT. Biochem J. 2016;473:2023.

13. (a) Aggarwal M, Kondeti B, McKenna R. Expert Opin Ther Pat. 2013;23:717; (b) Carta F, Supuran CT. Expert Opin Ther Pat. 2013;23:681;

(c) Thiry A, Dognè JM, Supuran CT, Masereel B. Curr Pharm Des. 2008;14:661; (d) Winum JY, Scozzafava A, Montero JL, Supuran CT. Med Res Rev. 2006;26:767;

(e) Di Fiore A, De Simone G, Alterio V, et al. Org Biomol Chem. 2016;14:4853.

14. (a) Arechederra RL, Waheed A, Sly WS, Supuran CT, Minteer SD. Bioorg Med Chem. 2013;21:1544;

(b) Scozzafava A, Supuran CT, Carta F. Expert Opin Ther Pat. 2013;23:725;

(c) Supuran CT. J Enzyme Inhib Med Chem. 2013;28:229.

15. (a) Supuran CT. Nat Rev Drug Discov. 2008;7:168;

(b) Ebbesen P, Pettersen EO, Gorr TA, et al. J Enzyme Inhib Med Chem. 2009;24:1; (c) Neri D, Supuran CT. Nat Rev Drug Discov. 2011;10:767.

16. (a) Supuran CT. Expert Rev Neurother. 2016;16:961;

(b) Carta F, Di Cesare Mannelli L, Pinard M, et al. Bioorg Med Chem. 2015;23:1828.

17. (a) Supuran CT. Bioorg Med Chem. 2013;21:1377;

(b) Supuran CT. J Enzyme Inhib Med Chem. 2016;31:345;

(c) Alterio V, Di Fiore A, D’Ambrosio K, Supuran CT, De Simone G. Chem Rev. 2012:112:4421.

18. Khalifah RG. J Biol Chem. 1971;246:2561.

19. (a) Gao BB, Clermont A, Rook S, et al. Nat Med. 2007;13:181; (b) Rose SJ, Bermudez LE. MBio. 2016;7:e01597.

20. (a) Datta R, Shah GN, Rubbelke TS, et al. Proc Natl Acad Sci USA. 2010;107:6448; (b) Ikeda Y, Yoshida N, Notomi S, et al. Br J Ophthalmol. 2013;97:1187.

21. (a) Lock FE, McDonald PC, Lou Y, et al. Oncogene. 2013;32:5210; (b) Bayram E, Senturk M, Kufrevioglu OI, Supuran CT. Bioorg Med Chem. 2008;16:9101:

(c) Vullo D, Voipio J, Innocenti A, et al. Bioorg Med Chem Lett. 2005;15:971.

22. De Simone G, Supuran CT. J Inorg Biochem. 2012;111:117.

23. (a) Korkmaz N, Obaidi OA, Senturk M, Astley D, Ekinci D, Supuran CT. J Enzyme Inhib Med Chem. 2015;30:75;

(b) Akdemir A, De Monte C, Carradori S, Supuran CT. J Enzyme Inhib Med Chem. 2015;30:114;

(c) Nishimori I, Vullo D, Innocenti A, Scozzafava A, Mastrolorenzo A, Supuran CT. J Med Chem. 2005;48:7860;

(d) Scozzafava A, Menabuoni L, Mincione F, Supuran CT. J Med Chem. 2002:45:1466.

24. (a) Sahin A, Isik S, Arslan O, Supuran CT, Ozensoy Guler O.J Enzyme Inhib Med Chem. 2015;30:224;

(b) Bozdag M, Isik S, Beyaztas S, Arslan O, Supuran CT. J Enzyme Inhib Med Chem. 2015;30:240;

(c) De Luca V, Del Prete S, Supuran CT, Capasso C. J Enzyme Inhib Med Chem. 2015;30:277;

(d) Vullo D, Milos M, Galic B, Scozzafava A, Supuran CT. J Enzyme Inhib Med Chem. 2015;30:341;

(e) Del Prete S, Vullo D, De Luca V, AlOthman Z, Supuran CT, Capasso C. J Enzyme Inhib Med Chem. 2015;30:366;

(f) Riafrecha LE, Vullo D, Supuran CT, Colinas PA. J Enzyme Inhib Med Chem. 2015;30:857.

25. (a) Göçer H, Akincioğlu AS, Gülçin GI, Supuran CT. J Enzyme Inhib Med Chem 2015;30:316;

(b) Ceruso M, Bragagni M, AlOthman Z, Osman SM, Supuran CT. J Enzyme Inhib Med Chem. 2015;30:430;

(c) Zolfaghari Emameh R, Syrjänen L, Barker H, Supuran CT, Parkkila S. J Enzyme Inhib Med Chem. 2015;30:505:

(d) Alafeefy AM, Ceruso M, Al-Tamimi AMS, Del Prete S, Supuran CT, Capasso C. J Enzyme Inhib Med Chem. 2015;30:592;

(e) Scozzafava A, Passaponti M, Supuran CT, Gülçin I. J Enzyme Med Chem. 2015;30:586;

(f) Le Darz A, Mingot A, Bouazza F, et al. J Enzyme Inhib Med Chem. 2015;30:737.

26. (a) Supuran CT, Barboiu M, Luca C, Pop E, Brewster ME, Dinculescu A. Eur J Med Chem. 1996;31:597-606:

(b) Carta F, Aggarwal M, Maresca A, et al. J Med Chem. 2012;55:1721-1730; (c) Puccetti L, Fasolis G, Vullo D, Chohan ZH, Scozzafava A, Supuran CT. Bioorg Med Chem Lett. 2005;15:3096-3101;

(d) Supuran CT, Nicolae A, Popescu A. Eur J Med Chem. 1996;31:431-438. 